\title{
The Adrenal Incidentaloma: An Opportunity to Improve Patient Care
}

\author{
James Becker, MD, Jakub Woloszyn, MD, Richard Bold, MD, and Michael J. Campbell, MD \\ Department of Surgery, University of California, Davis, Medical Center, Sacramento, CA, USA.
}

J Gen Intern Med 33(3):256-7

DOI: $10.1007 / \mathrm{s} 11606-017-4240-6$

(c) Society of General Internal Medicine 2017

\section{INTRODUCTION}

Increased recognition of the prevalence, dynamic nature, and biological significance of incidentally discovered adrenal tumors, or adrenal incidentalomas (AIs), has resulted in a growing clinical dilemma for primary care providers (PCPs). ${ }^{1,2}$ To help guide clinicians, the American Association of Endocrine Surgeons (AAES)/American Association of Clinical Endocrinologists (AACE) and, more recently, the European Society of Endocrinology have developed consensus guidelines to provide recommendations on the imaging, functional evaluation, and follow-up of patients with AIs. ${ }^{3,4}$ These guidelines recommend routine assessment for autonomous cortisol and catecholamine secretion for almost all patients, and selected evaluation of aldosterone secretion in hypertensive patients. Re-imaging to evaluate for malignancy, while controversial, should be considered in patients with indeterminate characteristics on initial imaging. ${ }^{4}$ The degree to which these guidelines have been integrated into routine practice by PCPs remains unclear. The purpose of this study is to evaluate adherence to the AACE/AAES guidelines for the management of AIs by PCPs and to assess their current management strategies.

\section{METHODS}

We reviewed the reports from 5063 consecutive abdominal CT scans for 4678 patients ordered by PCPs in the primary care network associated with our tertiary medical center from January 2011 to January 2014. The CT reports were searched for the following terms: adrenal mass, adrenal incidentaloma, adrenal lesion, adrenal tumor, adrenal adenoma, and adrenal nodule. Patients with known or clinically suspected adrenal lesions or active malignancy were excluded from evaluation.

The electronic charts of patients with an AI were reviewed for hormonal evaluation including plasma or urine metanephrines, 24-h urine cortisol or dexamethasone suppression test, and serum aldosterone-to-renin ratio (PAC:PRA) in hypertensive patients. Radiographic details including the size and non-contrast Hounsfield units of the tumor and use of reimaging were noted. Along with our chart review, we

Published online December 18, 2017 conducted an electronic survey of the PCPs who ordered the CT scans to better understand their approach to managing AIs. The two-tailed Fisher's exact test was used for bivariate analysis.

\section{RESULTS}

Of the 4678 patients reviewed, 209 (4.5\%) had an adrenal mass. Eighty-seven (1.9\%) patients met inclusion criteria and were included in our analysis. Of these 87 patients, $44(50.6 \%)$ were hypertensive, requiring medication. The baseline patient characteristics are summarized in Table 1.

Sixteen $(18.4 \%)$ patients had a complete initial hormonal evaluation that followed the AACE/AAES guidelines. Twenty-two $(25.3 \%)$ patients underwent a partial hormonal evaluation, including $20(23 \%)$ patients who had screening for pheochromocytoma, 19 (21.8\%) patients who had screening for hypercortisolism, and of the 44 hypertensive patients, 8 (18.2\%) who underwent PAC:PRA testing.

Most patients had a contrast-enhanced CT scan (57/87; $65.6 \%$ ). Of the 30 patients with non-contrast CTs, 19 had tumors that were $\leq 10 \mathrm{HU}$, suggesting a benign adenoma. Of the patients with indeterminate imaging (>10 HU), 46/67 $(69 \%)$ underwent re-imaging to evaluate for malignancy.

A standardized 16-question survey was sent to the 105 PCPs whose patients were included in the study, with 31 $(29.5 \%)$ responders. When asked about the significance of

Table 1 Baseline Characteristics of Patients Included in the Study $(N=87)$

\begin{tabular}{ll}
\hline \hline Indication for abdominal CT, $n(\%)$ & \\
Abdominal pain & $48(55.2)$ \\
Kidney stone evaluation & $19(21.8)$ \\
Nausea/Vomiting & $4(4.6)$ \\
Liver evaluation & $3(3.4)$ \\
Weight loss & $3(3.4)$ \\
Gastrointestinal bleeding & $2(2.3)$ \\
Hernia evaluation & $2(2.3)$ \\
Other & $6(6.9)$ \\
Non-contrast tumor attenuation, HU, mean (SD) & $14.6(21.3)$ \\
Bilateral incidentalomas, $n(\%)$ & $11(12.6)$ \\
Hypertensive, $n$ (\%) & $44(50.6)$ \\
Age, years, mean (SD) & $62.5(11.75)$ \\
BMI, kg/m 2 , mean (range) & $29.3(15.7-52.8)$ \\
Tumor size, mm, mean (range) & $15.5(5-37)$ \\
Included in CT impression, $n(\%)$ & $66(75.8)$ \\
\hline
\end{tabular}

CT, computed tomography; SD, standard deviation; HU, Hounsfield unit; BMI, body mass index 
Table 2 Association Between Likelihood of a Hormonal Evaluation and Mention of the Adrenal Tumor in the Impression of the Computed Tomography Report and Specialist Evaluation*

\begin{tabular}{|c|c|c|c|c|c|c|}
\hline & $\begin{array}{l}\text { Not reported } \\
\text { in CT impression }\end{array}$ & $\begin{array}{l}\text { Reported } \\
\text { in CT impression }\end{array}$ & $p$-value & Specialist evaluation & No specialist evaluation & $p$-value \\
\hline Cortisol evaluation & $0 / 21(0 \%)$ & $19 / 66(28.8 \%)$ & $<0.01$ & $10 / 12(83.3 \%)$ & $9 / 75(12 \%)$ & $<0.01$ \\
\hline Aldosterone evaluation & $0 / 12(0)$ & $8 / 32(25 \%)$ & 0.08 & $6 / 6(100 \%)$ & $2 / 38(5.3 \%)$ & $<0.01$ \\
\hline Catecholamine evaluation & $0 / 21(0 \%)$ & $20 / 66(30.3 \%)$ & $<0.01$ & $9 / 12(75 \%)$ & $11 / 75(14.7 \%)$ & $<0.01$ \\
\hline Complete evaluation & $0 / 21(0 \%)$ & $16 / 66(24.2 \%)$ & 0.02 & $9 / 12(75 \%)$ & $7 / 75(9.3 \%)$ & $<0.01$ \\
\hline
\end{tabular}

*Bivariate analysis by Fisher's exact test examining the association of outcomes with inclusion of an AI in the "Impression" section of the CT report and evaluation by a specialist

A $p$ value $<0.05$ was considered significant

CT, computed tomography

AIs, the majority of PCPs believed that most asymptomatic patients did not need a hormonal evaluation $(71 \%)$ or followup imaging (72\%). The majority $(60 \%)$ of respondents felt uncomfortable with the evaluation of AIs, yet $58 \%$ reported that it was their routine practice to conduct the work-up of AIs without specialist assistance.

Bivariate analysis demonstrated an association between reporting of the $\mathrm{AI}$ in the $\mathrm{CT}$ "Impression," and a complete assessment of hormonal secretion $(p=0.019)$. There was also a trend toward a specialist evaluation if the AI was mentioned in the "Impression," though it did not reach statistical significance $(p=0.06)$. Specialist evaluation was associated with increased likelihood of cortisol, aldosterone, and catecholamine evaluation $(p<0.01$; Table 2$)$.

\section{CONCLUSION}

Many patients with AIs are not evaluated for autonomous hormone production, which may lead to the underrecognition of potentially treatable disease. Further education and collaboration on the management of AIs in the primary care setting may provide an opportunity to improve patient care. We believe that our study reveals an opportunity for interdisciplinary care between endocrinologists, surgeons, and PCPs to address the potential for autonomous hormone production and the treatment options available to these patients.
Corresponding Author: Michael J. Campbell, MD; Department of Surgery University of California, Davis, Medical Center, Sacramento, CA, USA (e-mail: mjcampb@ucdavis.edu).

Funding The authors have received no external financial or material support related to this project.

\section{Compliance with Ethical Standards:}

Conflict of Interest: The authors declare that they have no conflict of interest.

\section{REFERENCES}

1. Kaltsas G, Chrisoulidou A, Piaditis G, Kassi E, Chrousos G. Current status and controversies in adrenal incidentalomas. Trends Endocrinol Metab. 2012;23:602-9.

2. Grumbach MM, Biller BM, Braunstein GD, Campbell KK, Carney JA, Godley PA, et al. Management of the clinically inapparent adrenal mass ("incidentaloma"). Ann Intern Med. 2003;138:424-9.

3. Zeiger MA, Thompson GB, Duh QY, Hamrahian AH, Angelos P, Elaraj D, et al. American Association of Clinical Endocrinologists and American Association of Endocrine Surgeons Medical Guidelines for the Management of Adrenal Incidentalomas: executive summary of recommendations. Endocr Pract. 2009; 15:450-3

4. Fassnacht M, Arlt W, Bancos I, Dralle H, Newell-Price J, Sahdev A, et al. Management of adrenal incidentalomas: European Society of Endocrinology Clinical Practice Guideline in collaboration with the European Network for the Study of Adrenal Tumors. Eur J Endocrinol. 2016;175(2):G1-G34. 